\title{
Ricci Curvature, Minimal Volumes, and Seiberg-Witten Theory
}

\author{
Claude LeBrun* \\ SUNY Stony Brook
}

March 9, 2000

\begin{abstract}
We derive new, sharp lower bounds for certain curvature functionals on the space of Riemannian metrics of a smooth compact 4manifold with a non-trivial Seiberg-Witten invariant. These allow one, for example, to exactly compute the infimum of the $L^{2}$-norm of Ricci curvature for all complex surfaces of general type. We are also able to show that the standard metric on any complex hyperbolic 4manifold minimizes volume among all metrics satisfying a point-wise lower bound on sectional curvature plus suitable multiples of the scalar curvature. These estimates also imply new non-existence results for Einstein metrics.
\end{abstract}

\section{Introduction}

The aim of modern Riemannian geometry is to understand the relationship between topology and curvature. It is thus interesting and natural to consider differential-topological invariants of a smooth compact $n$-manifold which, by their very definition, represent quantitative obstructions to the existence of a scalar-flat (or Ricci-flat, or flat) metric on the given manifold. For example, if $M$ is a smooth compact $n$-manifold, one may define invariants

$$
\mathcal{I}_{s}(M)=\inf _{g} \int_{M}\left|s_{g}\right|^{n / 2} d \mu_{g}
$$

\footnotetext{
${ }^{*}$ Supported in part by NSF grant DMS-9802722.
} 


$$
\begin{aligned}
\mathcal{I}_{r}(M) & =\inf _{g} \int_{M}|r|_{g}^{n / 2} d \mu_{g} \\
\mathcal{I}_{\mathcal{R}}(M) & =\inf _{g} \int_{M}|\mathcal{R}|_{g}^{n / 2} d \mu_{g}
\end{aligned}
$$

where the infima are to be taken over all metrics $g$ on $M$, where $s, r$, and $\mathcal{R}$ respectively denote the scalar, Ricci, and Riemann curvatures of $g$, and where $d \mu$ denotes Riemannian volume measure. The power of $n / 2$ is used for reason of scale invariance: any other choice would result in the zero invariant. Still, one has every right to suspect that invariants with such soft definitions will either be trivial, or else will be completely impossible to calculate in practice.

This suspicion would seem to be vindicated by a recent result of Petean [34], who, building upon the earlier work of Gromov-Lawson [17] and Stolz [38], showed that $\mathcal{I}_{s}(M)=0$ for any simply connected $n$-manifold, $n \geq 5$. Dimension 4, however, turns out to be radically different. Seiberg-Witten theory naturally leads to non-trivial lower bounds for $\mathcal{I}_{s}$ which, amazingly, are often sharp. Using this, the author has elsewhere 28, 29 computed $\mathcal{I}_{s}(M)$ for all complex surfaces $M$ with even first Betti number; it turns out that $\mathcal{I}_{s}(M)$ is positive exactly for the surfaces of general type, and for these it is given by the formula

$$
\mathcal{I}_{s}(M)=32 \pi^{2} c_{1}^{2}(X),
$$

where $X$ is the minimal model of $M$, in the sense of Kodaira.

One main purpose of the present article is to similarly calculate $\mathcal{I}_{r}$ for the complex surfaces. Unlike the $\mathcal{I}_{s}$, this invariant is changed by blowing up, and in Theorem 4.3 we will see that it is given by

$$
\mathcal{I}_{r}(M)=8 \pi^{2}\left[c_{1}^{2}(X)+k\right]
$$

where $k$ is the number of points of $X$ which must be blown up in order to obtain $M$.

This, however, is just one application of some new curvature estimates we will develop here. Fundamentally, the story is not about the Ricci curvature at all, but instead principally concerns the rôle of the self-dual Weyl curvature. Recall that the 2-forms on an oriented 4-manifold decompose as

$$
\Lambda^{2}=\Lambda^{+} \oplus \Lambda^{-},
$$


where $\Lambda^{ \pm}$is the $( \pm 1)$ eigenspace of Hodge star operator $\star$. Thinking of the curvature tensor $\mathcal{R}$ as a linear map $\Lambda^{2} \rightarrow \Lambda^{2}$, we thus get a decomposition 37

$$
\mathcal{R}=\left(\begin{array}{c|c}
W_{+}+\frac{s}{12} & B \\
\hline B^{*} & W_{-}+\frac{s}{12}
\end{array}\right)
$$

into irreducible pieces. Here the self-dual and anti-self-dual Weyl curvatures $W_{ \pm}$are the trace-free pieces of the appropriate blocks. The scalar curvature $s$ is understood to act by scalar multiplication, and $B$ amounts to the tracefree part $\stackrel{\circ}{r}=r-\frac{s}{4} g$ of the Ricci curvature, acting on anti-self-dual 2 -forms by

$$
\psi_{a b} \mapsto \stackrel{\circ}{r}_{a c} \psi_{b}^{c}-\stackrel{\circ}{r}_{b c} \psi_{a}^{c}{ }_{a} .
$$

The key estimate of the article is to be found in Theorem 2.3: if $(M, g)$ is an oriented Riemannian 4-manifold, and if, for a fixed $\operatorname{spin}^{c}$ structure on $M$, the Seiberg-Witten equations have a solution for every metric conformally related to $g$, then the curvature of $g$ satisfies

$$
\int_{M}\left(\frac{2}{3} s-2 \sqrt{\frac{2}{3}}\left|W_{+}\right|\right)^{2} d \mu \geq 32 \pi^{2}\left(c_{1}^{+}\right)^{2}
$$

where $c_{1}^{+}$is the self-dual part, with respect to $g$, of the first Chern class of the $\operatorname{spin}^{c}$ structure. Information about the Ricci curvature is then indirectly extracted from this, by means of the Gauss-Bonnet-like formula

$$
(2 \chi+3 \tau)(M)=\frac{1}{4 \pi^{2}} \int_{M}\left(2\left|W_{+}\right|^{2}+\frac{s^{2}}{24}-\frac{|\stackrel{\circ}{r}|^{2}}{2}\right) d \mu,
$$

which holds for every Riemannian metric $g$ on $M$. Here $\chi(M)$ and $\tau(M)$ denote the Euler characteristic and signature of $M$, respectively.

The above estimate should be compared and contrasted to the analogous inequality

$$
\int_{M} s^{2} d \mu \geq 32 \pi^{2}\left(c_{1}^{+}\right)^{2}
$$


for the scalar curvature, keeping in mind that $|s|=2 \sqrt{6}\left|W_{+}\right|$for any Kähler metric. This seems all the more remarkable insofar as the older estimate is only saturated by constant-scalar-curvature Kähler metrics, while the new estimate is apparently saturated by a larger class of almost-Kähler metrics. This reflects an under-utilized aspect of Taubes' construction of solutions of the Seiberg-Witten equations on symplectic manifolds [40, 41], and would appear to be a promising avenue for further research.

These estimates also give one new obstructions to the existence for Einstein metrics. Recall that that a smooth Riemannian metric $g$ is said to be Einstein if its Ricci curvature $r$ is a constant multiple of the metric:

$$
r=\lambda g
$$

Not every smooth compact oriented 4-manifold $M$ admits such a metric. Indeed, a well-known necessary condition is that $M$ must satisfy [19, 42, 60 the Hitchin-Thorpe inequality $2 \chi(M) \geq 3|\tau(M)|$, where again $\chi$ and $\tau$ denote the signature and Euler characteristic. Indeed, this is an immediate consequence of (11), since the Einstein condition may be rewritten as $\stackrel{\circ}{r}=0$, and $\stackrel{\circ}{r}$ makes the only negative contribution to the integrand. Notice, however, that one could strengthen the conclusion given an a priori lower bound for the term

$$
\frac{1}{4 \pi^{2}} \int_{M}\left(2\left|W_{+}\right|^{2}+\frac{s^{2}}{24}\right) d \mu .
$$

But a lower bound is not difficult to extract from the above estimate, and this allows one to show (Corollary 3.4) that if a minimal complex surface $X$ is blown up $k$ times, the resulting complex surface

$$
M=X \# k \overline{\mathbb{C P}}_{2}
$$

does not admit Einstein metrics if $k \geq \frac{1}{3} c_{1}^{2}(X)$. This improves previous results [28, 30], where the same conclusion is reached for larger values of $k$.

The invariant $\mathcal{I}_{\mathcal{R}}$ introduced at the beginning of this introduction is often easy to compute in dimension 4 , because [6]

$$
\mathcal{I}_{\mathcal{R}}(M) \geq 8 \pi^{2} \chi(M),
$$

with equality if $M$ admits an Einstein metric. In particular, it is trivial to read off this invariant for a compact hyperbolic 4 -manifold $\mathcal{H}^{4} / \Gamma$ or for a complex-hyperbolic 4-manifold $\mathbb{C} \mathcal{H}_{2} / \Gamma$. (The complex hyperbolic plane $\mathbb{C H}_{2}$ 
may abstractly be defined as the symmetric space $S U(2,1) / U(2)$, but it is typically more useful to think of it as the unit ball in $\mathbb{C}^{2}$, equipped with the Bergmann metric.) It might be tempting to assume that this tells one everything there is to know about the sectional curvatures of metrics on these spaces; but in reality, that is wide off the mark! For example, one might ask whether the standard metric, suitably normalized, has least volume among all metrics of sectional curvature $K \geq-1$. For real-hyperbolic manifolds the answer is affirmative, and indeed this holds in all dimensions; however, the proof, due to Besson-Courtois-Gallot [7], depends not only on a remarkable inequality concerning volume entropy, but also on an optimal application of Bishop's inequality. For complex-hyperbolic manifolds, the latter breaks down, and the question therefore remains open.

While the above question cannot be settled by present means, our estimates do allow one to answer a closely related question. Notice that a 4-manifold with $K \geq-1$ also satisfies

$$
\frac{1}{2}\left(K+\frac{s}{12}\right) \geq-1
$$

Given a complex hyperbolic 4-manifold, we are able to show (Theorem 5.3) that, among metrics on a complex-hyperbolic manifold satisfying this curvature constraint, an appropriate multiple of the standard metric has least volume. Similar results also hold metrics subject to the curvature constraint

$$
t K+(1-t) \frac{s}{12} \geq-1
$$

for any constant $t \in[0,1 / 2]$.

\section{Weyl Curvature Estimates}

In this section, we derive new $L^{2}$ estimates for combinations of the Weyl and scalar curvatures of certain Riemannian 4-manifolds. These considerably refine the estimates previously found in 30.

Let $M$ be a smooth, compact, oriented 4-manifold. Each Riemannian metric $g$ on $M$ then determines a direct sum decomposition

$$
H^{2}(M, \mathbb{R})=\mathcal{H}_{g}^{+} \oplus \mathcal{H}_{g}^{-},
$$

where $\mathcal{H}_{g}^{+}$(respectively, $\mathcal{H}_{g}^{-}$) consists of those cohomology classes for which the harmonic representative is self-dual (respectively, anti-self-dual). The 
non-negative integer $b_{+}(M)=\operatorname{dim} \mathcal{H}_{g}^{+}$is independent of $g$, and we will henceforth always assume it to be positive. It is thus natural to consider the set of metrics $g$ for which $\mathcal{H}_{g}^{+}=H$ for some fixed $b_{+}(M)$-dimensional subspace $H \subset H^{2}(M, \mathbb{R})$; such metrics will be said to be $H$-adapted. Assuming there is at least one $H$-adapted metric, we will then say that $H$ is a polarization of $M$, and call the pair $(M, H)$ a polarized 4-manifold [27]. Notice that the restriction of the intersection pairing

$$
\smile: H^{2}(M, \mathbb{R}) \times H^{2}(M, \mathbb{R}) \rightarrow \mathbb{R}
$$

to $H$ is then positive definite, and that $H \subset H^{2}$ is maximal among subspaces with this property.

Let $c$ be a $\operatorname{spin}^{c}$ structure on $M$. Then $c$ determines a Hermitian linebundle $L \rightarrow M$ with

$$
c_{1}(L) \equiv w_{2}(M) \bmod 2,
$$

and for each metric $g$ we also have rank-2 complex vector bundles $\mathbb{V}_{ \pm} \rightarrow M$ which formally satisfy

$$
\mathbb{V}_{ \pm}=\mathbb{S}_{ \pm} \otimes L^{1 / 2}
$$

where $\mathbb{S}_{ \pm}$are the locally-defined left- and right-handed spinor bundles of $g$. Given a polarization $H$ on $M$, we will then use $c_{1}^{+}$to denote the orthogonal projection of $c_{1}(L)$ into $H$ with respect to the intersection form. If $g$ is a particular metric with $\mathcal{H}_{g}^{+}=H$, we will also freely use $c_{1}(L)$ to denote the $g$-harmonic 2-form representing the corresponding de Rham class, and use $c_{1}^{+}$ to denote its self-dual part. For example, if a choice of $H$-compatible metric $g$ has already been made, the number

$$
\left|c_{1}^{+}\right|:=\sqrt{\left(c_{1}^{+}\right)^{2}}
$$

may freely be identified with the $\mathrm{L}^{2}$-norm of the self-dual $g$-harmonic form denoted by $c_{1}^{+}$.

For each Riemannian metric $g$, the Seiberg-Witten equations [45]

$$
\begin{aligned}
D_{A} \Phi & =0 \\
F_{A}^{+} & =i \sigma(\Phi)
\end{aligned}
$$

are equations for an unknown Hermitian connection $A$ on $L$ and an unknown smooth section $\Phi$ of $\mathbb{V}_{+}$. Here $D_{A}$ is the Dirac operator coupled to $A$, and $\sigma: \mathbb{V}_{+} \rightarrow \Lambda^{+}$is a certain canonical real-quadratic map. The latter formally 
arises from the fact that $\mathbb{V}_{+}=\mathbb{S}_{+} \otimes L^{1 / 2}$, whereas $\Lambda^{+} \otimes \mathbb{C}=\odot^{2} \mathbb{S}_{+}$; it is invariant under parallel transport, and satisfies $|\sigma(\Phi)|^{2}=|\Phi|^{4} / 8$. In conjunction with (3), the latter immediately gives us the important inequality

$$
\int_{M}|\Phi|^{4} d \mu \geq 32 \pi^{2}\left(c_{1}^{+}\right)^{2}
$$

because $2 \pi c_{1}^{+}$is the harmonic part of $-\sigma(\Phi)$.

In this paper, we will primarily be interested in 4-manifolds for which there is a solution of the Seiberg-Witten equations for each metric. Let us make this more precise by introducing some terminology; cf. 25.

Definition 1 Let $M$ be a smooth compact oriented 4-manifold, let $H \subset$ $H^{2}(M, \mathbb{R})$ be a polarization of $M$, and let c be a spinc structure on $M$. Then we will say that $c$ is a monopole class for $(M, H)$ if the Seiberg-Witten equations (2-3) have a solution for every $H$-adapted metric $g$.

This definition will be useful in practice, of course, only because of the existence of Seiberg-Witten invariants 24, 45]. For example, if a $\operatorname{spin}^{c}$ structure satisfies $\left[c_{1}(L)\right]^{2}=(2 \chi+3 \tau)(M)$, and if $c_{1}^{+} \neq 0$ relative to the polarization $H=\mathcal{H}_{g}^{+}$, then the Seiberg-Witten invariant $\mathcal{S} \mathcal{W}_{c}(M ; H)$ can be defined as the number of solutions, modulo gauge transformations and counted with orientations, of a generic perturbation

$$
\begin{aligned}
D_{A} \Phi & =0 \\
i F_{A}^{+}+\sigma(\Phi) & =\phi
\end{aligned}
$$

of (2-3), where $\phi$ is a smooth self-dual 2-form of small $L^{2}$ norm. If this invariant is non-zero, then $c$ is a monopole class for $(M, H)$, where $H$ is the polarization determined by the metric $g$. If $b^{+}(M) \geq 2, \mathcal{S} W_{c}(M, H)$ is actually independent of the polarization $H$; when $b^{+}(M)=1$, by contrast, it is well defined only for those polarizations for which $c_{1}^{+} \neq 0$, and its value typically depends on whether $c_{1}^{+}$is a future-pointing or past-pointing timelike vector in the Lorentzian vector space $H^{2}(M, \mathbb{R})$.

More generally [32, 31], suppose that we have a $\operatorname{spin}^{c}$ structure such that

$$
\ell=\frac{\left[c_{1}(L)\right]^{2}-(2 \chi+3 \tau)(M)}{4}
$$

is a non-negative integer. Then the moduli space $\mathcal{M}_{c, g}$ of gauge-equivalence classes of solutions of a generic perturbation of (2-3) is a smooth compact 
$\ell$-manifold; moreover, it acquires a canonical orientation once we choose an orientation for the vector space $H^{1}(M, \mathbb{R}) \oplus H$. Fix $\ell$ loops $\beta_{1}, \ldots, \beta_{\ell}$ in $M$, and define a smooth map $\mathcal{M}_{c, g} \rightarrow T^{\ell}$ from the moduli space to the $\ell$-torus by sending the gauge-equivalence class $[(\Phi, A)]$ to the holonomies of the $U(1)$ connection $A$ around the $\ell$ given loops. The homotopy class of this map only depends on the homology classes $\left[\beta_{i}\right] \in H_{1}(M, \mathbb{Z})$, one we may therefore define $\mathcal{S} \mathcal{W}_{c}\left(M,\left[\beta_{1}\right], \cdots,\left[\beta_{\ell}\right] ; H\right) \in \mathbb{Z}$ to be the degree of this map. Again, if this invariant is non-zero, $c$ is a monopole class of $(M, H)$.

Many of the most remarkable consequences of Seiberg-Witten theory stem from the fact that the equations (2) imply the Weitzenböck formula

$$
0=4 \nabla^{*} \nabla \Phi+s \Phi+|\Phi|^{2} \Phi
$$

where $s$ denotes the scalar curvature of $g$. Taking the inner product with $\Phi$, it follows that

$$
0=2 \Delta|\Phi|^{2}+4|\nabla \Phi|^{2}+s|\Phi|^{2}+|\Phi|^{4} .
$$

If we multiply (6) by $|\Phi|^{2}$ and integrate, we have

$$
0=\int_{M}\left[\left.\left.2|d| \Phi\right|^{2}\right|^{2}+4|\Phi|^{2}|\nabla \Phi|^{2}+s|\Phi|^{4}+|\Phi|^{6}\right] d \mu_{g}
$$

so that

$$
\int(-s)|\Phi|^{4} d \mu \geq 4 \int|\Phi|^{2}|\nabla \Phi|^{2} d \mu+\int|\Phi|^{6} d \mu .
$$

We will see in a moment that this implies some remarkable estimates for the Weyl curvature of suitable 4-manifolds.

Before doing so, however, let us first recall that the self-dual Weyl tensor at a point $x$ of an oriented Riemannian 4-manifold $(M, g)$ may be viewed as a trace-free endomorphism $W_{+}(x): \Lambda_{x}^{+} \rightarrow \Lambda_{x}^{+}$of the self-dual 2 -forms at $x$. We will use $w(x)$ denotes its lowest eigenvalue; notice that this is automatically a Lipschitz continuous function $w: M \rightarrow(-\infty, 0]$. Let us also fix the notational convention that, for any real-valued function $f: M \rightarrow \mathbb{R}, f_{-}$: $M \rightarrow(-\infty, 0]$ is defined by $f_{-}(x)=\min (f(x), 0)$. With these preliminaries, we are now prepared to prove our first, crucial result:

Proposition 2.1 Let $(M, g)$ be a compact oriented Riemannian 4-manifold on which there is a solution of the Seiberg-Witten equations. Let $c_{1}(L)$ be the 
first Chern class of the relevant spinc ${ }^{c}$ structure, and let $c_{1}^{+}$denote its self-dual part with respect to $\mathrm{g}$. Then

$$
V^{1 / 3}\left(\int_{M}\left|\left(\frac{2}{3} s_{g}+2 w_{g}\right)_{-}\right|^{3} d \mu\right)^{2 / 3} \geq 32 \pi^{2}\left(c_{1}^{+}\right)^{2},
$$

where $V=\operatorname{Vol}(M, g)=\int_{M} d \mu_{g}$ is the total volume of $(M, g)$.

Proof. Any self-dual 2-form $\psi$ on any oriented 4-manifold satisfies the Weitzenböck formula [9]

$$
\left(d+d^{*}\right)^{2} \psi=\nabla^{*} \nabla \psi-2 W_{+}(\psi, \cdot)+\frac{s}{3} \psi,
$$

where $W_{+}$is the self-dual Weyl tensor. It follows that

$$
\int_{M}\left(-2 W_{+}\right)(\psi, \psi) \geq \int_{M}\left(-\frac{s}{3}\right)|\psi|^{2} d \mu-\int_{M}|\nabla \psi|^{2} d \mu,
$$

so that

$$
-\int_{M} 2 w|\psi|^{2} \geq \int_{M}\left(-\frac{s}{3}\right)|\psi|^{2} d \mu-\int_{M}|\nabla \psi|^{2} d \mu,
$$

and hence

$$
-\int_{M}\left(\frac{2}{3} s+2 w\right)|\psi|^{2} \geq \int_{M}(-s)|\psi|^{2} d \mu-\int_{M}|\nabla \psi|^{2} d \mu .
$$

On the other hand, the particular self-dual 2-form $\varphi=\sigma(\Phi)=-i F_{A}^{+}$satisfies

$$
\begin{aligned}
|\varphi|^{2} & =\frac{1}{8}|\Phi|^{4}, \\
|\nabla \varphi|^{2} & \leq \frac{1}{2}|\Phi|^{2}|\nabla \Phi|^{2} .
\end{aligned}
$$

Setting $\psi=\varphi$, we thus have

$$
-\int_{M}\left(\frac{2}{3} s+2 w\right)|\Phi|^{4} \geq \int_{M}(-s)|\Phi|^{4} d \mu-4 \int_{M}|\Phi|^{2}|\nabla \Phi|^{2} d \mu .
$$

But (7) tells us that

$$
\int_{M}(-s)|\Phi|^{4} d \mu-4 \int_{M}|\Phi|^{2}|\nabla \Phi|^{2} d \mu \geq \int_{M}|\Phi|^{6} d \mu,
$$


so we obtain

$$
-\int_{M}\left(\frac{2}{3} s+2 w\right)_{-}|\Phi|^{4} d \mu \geq-\int_{M}\left(\frac{2}{3} s+2 w\right)|\Phi|^{4} d \mu \geq \int_{M}|\Phi|^{6} d \mu .
$$

By the Hölder inequality, we thus have

$$
\left(\int\left|\left(\frac{2}{3} s+2 w\right)_{-}\right|^{3} d \mu\right)^{1 / 3}\left(\int|\Phi|^{6} d \mu\right)^{2 / 3} \geq \int|\Phi|^{6} d \mu
$$

Since the Hölder inequality also tells us that

$$
\int|\Phi|^{6} d \mu \geq V^{-1 / 2}\left(\int|\Phi|^{4} d \mu\right)^{3 / 2}
$$

we thus have

$$
V^{1 / 3}\left(\int_{M}\left|\left(\frac{2}{3} s_{g}+2 w_{g}\right)_{-}\right|^{3} d \mu\right)^{2 / 3} \geq \int|\Phi|^{4} d \mu \geq 32 \pi^{2}\left(c_{1}^{+}\right)^{2},
$$

as claimed.

While this result is the key to everything that follows, its direct utility is limited by the fact that it is an $L^{3}$, rather than an $L^{2}$, estimate. Fortunately, however, we will be able to extract an $L^{2}$ estimate by means of a conformal rescaling trick, the general idea of which is drawn from Gursky [18]:

Lemma 2.2 Let $(M, \gamma)$ be a compact oriented 4-manifold with a fixed smooth conformal class of Riemannian metrics. Suppose, moreover, that $\gamma$ does not contain a metric of positive scalar curvature. Then, for any $\alpha \in(0,1)$, there is a metric $g_{\gamma} \in \gamma$ of differentiability class $C^{2, \alpha}$ for which $s+3 w$ is a nonpositive constant.

Proof. Let $g_{0} \in \gamma$ be a fixed smooth back-ground metric, and notice that, for $g=u^{2} g_{0} \in \gamma$, the function $\mathfrak{S}_{g}=s_{g}+3 w_{g}$ is given by

$$
\mathfrak{S}_{g}=u^{-3}\left(6 \Delta_{g_{0}} u+\mathfrak{S}_{g_{0}} u\right)
$$

by virtue of the weighted conformal invariance of $W_{+}$. Thus $\mathfrak{S}$ behaves under conformal rescaling just like the scalar curvature, despite the fact that 
it might well only be a Lipschitz function. In order to find a suitable choice of $u$, we therefore attempt to minimize

$$
\mathcal{F}(u)=\frac{\int_{M}\left(6|d u|^{2}+\mathfrak{S}_{g_{0}} u^{2}\right) d \mu_{g_{0}}}{\left(\int_{M} u^{4} d \mu_{g_{0}}\right)^{1 / 2}}
$$

on the positive sector of the unit sphere in the Sobolev space $L_{1}^{2}\left(M, g_{0}\right)$. Yamabe's ansatz for doing this [46, 4] is to minimize the functionals

$$
\mathcal{F}_{\epsilon}(u)=\frac{\int_{M}\left(6|d u|^{2}+\mathfrak{S}_{g_{0}} u^{2}\right) d \mu_{g_{0}}}{\left(\int_{M} u^{4-\epsilon} d \mu_{g_{0}}\right)^{1 /\left(2-\frac{\epsilon}{2}\right)}}
$$

and then take the limit of the minimizers as $\epsilon \searrow 0$. For each $\epsilon>0$, the existence of minimizers follows from the Sobolev embedding theorem; indeed,

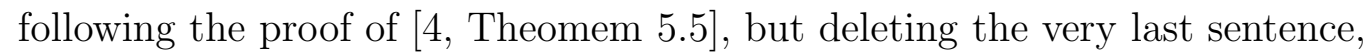
one obtains a positive function $u_{\epsilon}$ of class $C^{2, \alpha}$ which solves

$$
6 \Delta_{g_{0}} u+\mathfrak{S}_{g_{0}} u=c_{\epsilon} u^{3-\epsilon}
$$

where $c_{\epsilon}$ is the infimum of $\mathcal{F}_{\epsilon}$ on the positive sector of the unit sphere of $L_{1}^{2}\left(M, g_{0}\right)$. The convergence as $\epsilon \searrow 0$ then follows from an observation of Trudinger [43. Namely, since $\mathfrak{S} \leq s$ for any metric, and since there is nothing to prove if $\mathfrak{S}=s$, our hypothesis that there is no metric of positive scalar curvature in $\gamma$ allows us to assume that that $c_{0}=\inf c_{\epsilon}<0$. Inspection of (9) at a maximum then gives us the $C^{0}$ estimate

$$
u_{\epsilon} \leq 1+\sqrt{\left|\frac{\inf \mathfrak{S}_{g_{0}}}{c_{0}}\right|}
$$

for all small $\varepsilon$. This implies [4, Theorem 6.5] that $u=\lim _{\epsilon \rightarrow 0} u_{\epsilon}$ exists in $C^{1}$, and is a weak solution of

$$
6 \Delta_{g_{0}} u+\mathfrak{S}_{g_{0}} u=c_{0} u^{3}
$$

Schauder theory then tells us that $u$ is actually a $C^{2, \alpha}$ function. In particular, $g_{\gamma}=u^{2} g_{0}$ is a $C^{2, \alpha}$ metric, with curvature satisfying $\mathfrak{S}_{g_{\gamma}}=c_{0}$ in the classical sense. 
Theorem 2.3 Let $(M, H)$ be a polarized smooth compact oriented 4manifold, and let $c$ be a monopole class for $(M, H)$. Let $c_{1}(L) \in H^{2}(M, \mathbb{R})$ denote the anti-canonical class of $c$, and let $c_{1}^{+} \neq 0$ be its orthogonal projection to $H$ with respect to the intersection form. Then every $H$-adapted Riemannian metric $g$ satisfies

$$
\int_{M}\left(\frac{2}{3} s-2 \sqrt{\frac{2}{3}}\left|W_{+}\right|\right)^{2} d \mu \geq 32 \pi^{2}\left(c_{1}^{+}\right)^{2} .
$$

Proof. Let $\gamma$ be the conformal class of some $H$-adapted metric $g$. Since the Hodge star operator is conformally invariant in the middle dimension, every metric in $\gamma$ is also $H$-adapted. Since $c$ is a monopole class for $H$, it therefore follows that $\gamma$ does not contain any metrics of positive scalar curvature. Lemma 2.2 therefore applies, and tells us that the conformal class $\gamma$ contains a metric $g_{\gamma}$ for which that $\frac{2}{3} s+2 w=\frac{2}{3} \mathfrak{S}$ is a non-positive constant. For this metric, one then has

$$
\int_{M}\left|\left(\frac{2}{3} s_{g_{\gamma}}+2 w_{g_{\gamma}}\right)_{-}\right|^{2} d \mu_{g_{\gamma}}=V_{g_{\gamma}}^{1 / 3}\left(\int_{M}\left|\left(\frac{2}{3} s_{g_{\gamma}}+2 w_{g_{\gamma}}\right)_{-}\right|^{3} d \mu_{g_{\gamma}}\right)^{2 / 3}
$$

so that

$$
\int_{M}\left|\left(\frac{2}{3} s_{g_{\gamma}}+2 w_{g_{\gamma}}\right)_{-}\right|^{2} d \mu_{g_{\gamma}} \geq 32 \pi^{2}\left(c_{1}^{+}\right)^{2}
$$

by Proposition 2.1. Thus we at least have an $L^{2}$ estimate concerning the conformally related metric $g_{\gamma}$.

Let us now compare the left-hand side with analogous expression for the given metric $g$. To do so, we express $g$ in the form $g=u^{2} g_{\gamma}$, where $u$ is a positive $C^{2}$ function, and observe that

$$
\begin{aligned}
\int_{M}\left(\frac{2}{3} s_{g}+2 w_{g}\right)_{-} u^{2} d \mu_{g_{\gamma}} & \leq \int_{M}\left(\frac{2}{3} s_{g}+2 w_{g}\right) u^{2} d \mu_{g_{\gamma}} \\
& =\frac{2}{3} \int \mathfrak{S}_{g} u^{2} d \mu_{g_{\gamma}} \\
& =\frac{2}{3} \int u^{-3}\left(6 \Delta_{g_{\gamma}} u+\mathfrak{S}_{g_{\gamma}} u\right) u^{2} d \mu_{g_{\gamma}} \\
& =\frac{2}{3} \int\left(-6 u^{-2}|d u|_{g_{\gamma}}^{2}+\mathfrak{S}_{g_{\gamma}}\right) d \mu_{g_{\gamma}}
\end{aligned}
$$




$$
\begin{aligned}
& \leq \frac{2}{3} \int \mathfrak{S}_{g_{\gamma}} d \mu_{g_{\gamma}} \\
& =\int_{M}\left(\frac{2}{3} s_{g_{\gamma}}+2 w_{g_{\gamma}}\right)_{-} d \mu_{g_{\gamma}} .
\end{aligned}
$$

Applying Cauchy-Schwarz, we thus have

$$
\begin{aligned}
-V_{g_{\gamma}}^{1 / 2}\left(\int\left|\left(\frac{2}{3} s_{g}+2 w_{g}\right)_{-}\right|^{2} d \mu_{g}\right)^{1 / 2} & =-V_{g_{\gamma}}^{1 / 2}\left(\int\left(\frac{2}{3} s_{g}+2 w_{g}\right)^{2} u^{4} d \mu_{g_{\gamma}}\right)^{1 / 2} \\
& \leq \int_{M}\left(\frac{2}{3} s_{g}+2 w_{g}\right) u^{2} d \mu_{g_{\gamma}} \\
& \leq \int_{M}\left(\frac{2}{3} s_{g_{\gamma}}+2 w_{g_{\gamma}}\right) d \mu_{g_{\gamma}} \\
& =-V_{g_{\gamma}}^{1 / 2}\left(\int\left|\left(\frac{2}{3} s_{g_{\gamma}}+2 w_{g_{\gamma}}\right)_{-}\right|^{2} d \mu_{g_{\gamma}}\right)^{1 / 2}
\end{aligned}
$$

and hence

$$
\int_{M}\left|\left(\frac{2}{3} s_{g}+2 w_{g}\right)_{-}\right|^{2} d \mu_{g} \geq \int_{M}\left|\left(\frac{2}{3} s_{g_{\gamma}}+2 w_{g_{\gamma}}\right)_{-}\right|^{2} d \mu_{g_{\gamma}} .
$$

This shows that

$$
\int_{M}\left|\left(\frac{2}{3} s_{g}+2 w_{g}\right)_{-}\right|^{2} d \mu_{g} \geq 32 \pi^{2}\left(c_{1}^{+}\right)^{2}
$$

for every $H$-adapted metric $g$.

Finally, we observe that

$$
-\sqrt{\frac{2}{3}}\left|W_{+}\right|_{g} \leq w_{g}
$$

for any metric, simply because $W_{+}$is trace-free. It follows that

$$
\left(\frac{2}{3} s-2 \sqrt{\frac{2}{3}}\left|W_{+}\right|_{g}\right)^{2} \geq\left|\left(\frac{2}{3} s_{g}+2 w_{g}\right)_{-}\right|^{2}
$$

at every point, and (11) therefore implies the desired inequality (10). 
The inequalities we have just derived are certainly sharp, in the sense that equality is attained by any Kähler metric of constant negative scalar curvature. However, we will now see that equality also holds in principle for a broader class of metrics. To make this precise, suppose that $(M, \omega)$ is a symplectic 4-manifold, and let $g$ be a Riemannian metric such that

$$
\omega(\cdot, \cdot)=g(J \cdot, \cdot)
$$

for some almost complex structure $J$ on $M$; equivalently, suppose that $\omega$ is self-dual with respect to $g$ and has constant norm $|\omega|_{g}=\sqrt{2}$. One then says that $g$ is an almost-Kähler metric, with almost-Kähler form $\omega$. Evidently, $g$ is actually Kähler if $J$ is integrable, but Gromov's theory of pseudo-holomorphic curves [16] and Taubes' characterization [40, 41] of the Seiberg-Witten invariants of symplectic 4-manifolds have conclusively swept the study of such metrics into the mathematical main-stream.

In order to state our next result, we will need extra terminology in this connection. The *-scalar curvature of a 4-dimensional almost-Kähler manifold $(M, g, \omega)$ is by definition the function

$$
s^{*}=\frac{s}{3}+2 W_{+}(\omega, \omega) .
$$

This definition is motivated by the fact that the scalar curvature $s$ and *scalar curvature $s^{*}$ coincide for any Kähler manifold. Notice that we automatically have the inequality

$$
s^{*} \geq \frac{s}{3}+4 w
$$

with equality iff the almost-Kähler form $\omega$ belongs to the lowest eigenspace of $W_{+}$.

Proposition 2.4 Suppose that equality holds in either (8) or (11). Then $g$ is an almost-Kähler metric with the following properties:

- the almost-Kähler form $\omega$ belongs, at each $x \in M$, to the lowest eigenspace of $W_{+}$;

- the sum $s+s^{*}$ of the scalar and $*$-scalar curvatures is a non-positive constant; 
- the first Chern class of $(M, \omega)$ is of type $(1,1)$, and coincides with first Chern class $c_{1}(L)$ of the relevant spin ${ }^{c}$ structure.

If equality holds in (10), these same conclusions hold, but, in addition,

- the self-dual Weyl curvature $W_{+}$is invariant with respect to the almostcomplex structure $J$ of $(M, g, \omega)$.

Conversely, any compact almost-Kähler 4-manifold satisfying these conditions saturates the corresponding inequalities, and its first Chern class is a monopole class of the corresponding polarization.

Proof. If equality holds in (8), the last inequality in the proof of Proposition 2.1 forces $\sigma(\Phi)$ to be the harmonic representative of $-2 \pi c_{1}^{+}$. Moreover, the Hölder inequalities used in that proof shows that equality can only hold if $|\Phi|^{2}$ and $-\left(\frac{2}{3} s+2 w\right)$ are constant, and moreover equal. Thus the harmonic form $\varphi=\sigma(\Phi)$ has constant norm, so that $\omega=-\sqrt{2} \varphi /|\varphi|$ is an almostKähler form compatible with $g$. Moreover, $L=\operatorname{det}\left(\mathbb{V}_{+}\right)=\mathbb{V}_{+} / \operatorname{span}(\Phi)$ coincides with the anti-canonical line bundle associated with the almostcomplex structure $J$. Since $c_{1}^{+}$is now a (non-positive) multiple of $[\omega]$ and since the volume $V=\operatorname{Vol}(M, g)$ of an almost-Kähler 4-manifold is exactly $[\omega]^{2} / 2$, the fact that $\frac{2}{3} s+2 w$ is a non-positive constant tells us that we now have

$$
\int\left(\frac{2}{3} s+2 w\right) d \mu=-V^{1 / 2} 4 \sqrt{2} \pi\left|c_{1}^{+}\right|=4 \pi c_{1}(L) \cdot[\omega],
$$

since by assumption equality holds in (8). It thus follows that

$$
\int \frac{1}{2}\left(s+s^{*}\right) d \mu \geq 4 \pi c_{1}(L) \cdot[\omega],
$$

with equality iff $\omega$ everywhere belongs to the lowest eigenspace of $W_{+}$. However, Blair [8] has shown that

$$
\int \frac{1}{2}\left(s+s^{*}\right) d \mu=4 \pi c_{1} \cdot[\omega]
$$

for any almost-Kähler 4-manifold. We thus conclude that $\omega$ is everywhere in the lowest eigenspace of $W_{+}$.

If we instead have equality in (11) or (10), we must in particular be at the minimum of

$$
\int\left|\left(\frac{2}{3} s+2 w\right)_{-}\right|^{2} d \mu
$$


among metrics in the given conformal class. This then implies that $\frac{2}{3} s+2 w$ is constant, and we therefore also have equality in (8), and the claim follows from the previous argument.

The converse assertions follow from [40] and (12). Details are left to the interested reader.

In a naïve sense, this provides a complete characterization of the metrics which saturate (8), (10), and (11). It remains to be seen, however, whether such metrics can ever be strictly almost-Kähler, in the sense that the almostcomplex structure $J$ fails to be integrable. For example, a beautiful recent result of J. Armstrong [2] asserts the non-existence of compact, Einstein, strictly almost-Kähler 4-manifolds on which $\omega$ is everywhere an eigenvector of $W_{+}$. Thus:

Corollary 2.5 Suppose that $g$ is an Einstein metric which saturates (8), (10), or (11). Then $g$ is actually Kähler-Einstein.

Similarly, a recent result of Apostolov-Armstrong-Drăghici [1] implies that an almost-Kähler metric saturating (10) is Kähler iff its Ricci tensor is $J$-invariant.

\section{Einstein Metrics}

Our first application of the preceding Weyl estimates will be to prove new non-existence for Einstein metrics on suitable smooth compact 4-manifolds. We begin by proving the following technical result:

Proposition 3.1 Let $(M, H)$ be a polarized smooth compact oriented 4manifold with monopole class $c$. Then every $H$-adapted metric satisfies

$$
\frac{1}{4 \pi^{2}} \int_{M}\left(\frac{s_{g}^{2}}{24}+2\left|W_{+}\right|_{g}^{2}\right) d \mu_{g} \geq \frac{2}{3}\left(c_{1}^{+}\right)^{2} .
$$

If equality holds, moreover, $g$ is an almost-Kähler metric of the type described in Proposition 2.4.

Proof. Let us begin by rewriting the inequality (10) as

$$
\left\|\frac{2}{3} s-2 \sqrt{\frac{2}{3}}\left|W_{+}\right|\right\| \geq 4 \sqrt{2} \pi\left|c_{1}^{+}\right|,
$$


where $\|\cdot\|$ denotes the $L^{2}$ norm with respect to $g$. By the triangle inequality, we therefore have

$$
\frac{2}{3}\|s\|+\frac{1}{3}\left\|\left(\sqrt{24}\left|W_{+}\right|\right)\right\| \geq 4 \sqrt{2} \pi\left|c_{1}^{+}\right|
$$

We now elect to interpret the left-hand side as the dot product

$$
\left(\frac{2}{3}, \frac{1}{3 \sqrt{2}}\right) \cdot\left(\|s\|,\left\|\left(\sqrt{48}\left|W_{+}\right|\right)\right\|\right)=\frac{2}{3}\|s\|+\frac{1}{3}\left\|\left(\sqrt{24}\left|W_{+}\right|\right)\right\|
$$

in $\mathbb{R}^{2}$. Applying Cauchy-Schwarz, we thus have

$$
\left(\left(\frac{2}{3}\right)^{2}+\left(\frac{1}{3 \sqrt{2}}\right)^{2}\right)^{1 / 2}\left(\int_{M}\left(s^{2}+48\left|W_{+}\right|^{2}\right) d \mu\right)^{1 / 2} \geq \frac{2}{3}\|s\|+\frac{1}{3}\left\|\left(\sqrt{24}\left|W_{+}\right|\right)\right\| .
$$

Thus

$$
\frac{1}{2} \int_{M}\left(s^{2}+48\left|W_{+}\right|^{2}\right) d \mu \geq 32 \pi^{2}\left(c_{1}^{+}\right)^{2}
$$

so that

$$
\frac{1}{4 \pi^{2}} \int_{M}\left(\frac{s_{g}^{2}}{24}+2\left|W_{+}\right|_{g}^{2}\right) d \mu_{g} \geq \frac{2}{3}\left(c_{1}^{+}\right)^{2}
$$

as claimed. If equality holds, moreover, (10) is saturated, and Proposition 2.4 applies.

Proposition 3.2 Let $X$ be a compact oriented 4-manifold with a non-trivial Seiberg-Witten invariant, and set

$$
M=X \# k \overline{\mathbb{C P}}_{2} \# \ell\left(S^{1} \times S^{3}\right)
$$

for integers $k, \ell \geq 0$. Then any Riemannian metric $g$ on $M$ satisfies

$$
\frac{1}{4 \pi^{2}} \int_{M}\left(\frac{s_{g}^{2}}{24}+2\left|W_{+}\right|_{g}^{2}\right) d \mu_{g} \geq \frac{2}{3}(2 \chi+3 \tau)(X),
$$

and equality is possible only if $k=\ell=0$.

Proof. The proof is a direct extension of the computations in [30], although for $\ell>0$ we now use the holonomy-constrained Seiberg-Witten invariant of [32]; cf. [11, 31]. 
Now notice that we may assume that $(2 \chi+3 \tau)(X)>0$, since otherwise there is nothing to prove. This has the pleasant consequence that any Seiberg-Witten invariant of $X$ is independent of polarization, even if $b^{+}(X)=1$. Let $c_{1}(X)$ denote the first Chern class of a $\operatorname{spin}^{c}$ structure on $X$ for which the Seiberg-Witten invariant is non-zero, and notice that $\left(c_{1}(X)\right)^{2} \geq(2 \chi+3 \tau)(X)>0$, because the relevant Seiberg-Witten moduli space must have non-negative virtual dimension. Pull $c_{1}(X)$ back to $M=X \# k \overline{\mathbb{C P}}_{2} \ell\left(S^{1} \times S^{3}\right)$ via the canonical collapsing map, and, by a standard abuse of notation, let $c_{1}(X)$ also denote this pulled-back class. Thus, with respect to our given polarization,

$$
\left(\left[c_{1}(X)\right]^{+}\right)^{2} \geq\left(c_{1}(X)\right)^{2} \geq(2 \chi+3 \tau)(X)>0 .
$$

Now choose generators $E_{1}, \ldots, E_{k}$ for the pull-backs to $M$ of the $k$ relevant copies of $H^{2}\left(\overline{\mathbb{C P}}_{2}, \mathbb{Z}\right)$ so that

$$
\left[c_{1}(X)\right]^{+} \cdot E_{j} \leq 0, \quad j=1, \ldots, k .
$$

Let $\beta_{1}, \ldots, \beta_{\ell}$ be closed curves in $M$ which generate of the fundamental groups of the $\ell$ relevant copies of $S^{1} \times S^{3}$. Then [14, 32] there is a $\operatorname{spin}^{c}$ structure on $M$ with $\mathcal{S W}_{c}\left(M,\left[\beta_{1}\right], \cdots,\left[\beta_{\ell}\right] ; H\right) \neq 0$ and

$$
c_{1}(L)=c_{1}(X)-\sum_{j=1}^{k} E_{j} .
$$

Thus $c$ is a monopole class of $(M, H)$. But one then has

$$
\begin{aligned}
\left(c_{1}^{+}\right)^{2} & =\left(\left[c_{1}(X)\right]^{+}+\sum_{j=1}^{k} E_{j}^{+}\right)^{2} \\
& =\left(\left[c_{1}(X)\right]^{+}\right)^{2}-2 \sum_{j=1}^{k}\left[c_{1}(X)\right]^{+} \cdot E_{j}+\left(\sum_{j=1}^{k} E_{j}^{+}\right)^{2} \\
& \geq\left(\left[c_{1}(X)\right]^{+}\right)^{2} \\
& \geq(2 \chi+3 \tau)(X),
\end{aligned}
$$

exactly as claimed.

If equality held, $g$ would be almost-Kähler, and $c_{1}(L)$ would be the anticanonical class of the associated almost-complex structure on $M$. But, by construction,

$$
c_{1}^{2}(L)=\left(c_{1}(X)-\sum E_{j}\right)^{2}=(2 \chi+3 \tau)(X)-k,
$$


whereas

$$
(2 \chi+3 \tau)(M)=(2 \chi+3 \tau)(X)-k-4 \ell
$$

by Mayer-Vietoris. Since $c_{1}^{2}=2 \chi+3 \tau$ for an almost-complex manifold, equality is thus excluded unless $\ell=0$.

There is yet more information available, however. Indeed, if equality held, the almost-Kähler class $[\omega]$ would also necessarily be a non-positive multiple of $c_{1}^{+}$. On the other hand, our computation shows that equality can only hold if $\left[c_{1}(X)\right]^{+} \cdot E_{j}=0$, so it would follow that $[\omega] \cdot E_{j}=0$ for all $j$. However, the Seiberg-Witten invariant would then also be non-trivial for a $\operatorname{spin}^{c}$ structure with $c_{1}(\tilde{L})=c_{1}(L)+2 E_{1}$, and a celebrated theorem of Taubes [41] would then force the homology class $E_{j}$ to be represented by a pseudo-holomorphic 2-sphere in the symplectic manifold $(M, \omega)$. But the (positive!) area of this sphere with respect to $g$ would then be exactly $[\omega] \cdot E_{j}$, contradicting the observation that $[\omega] \cdot E_{j}=0$. Thus equality can definitely be excluded unless $k$ and $\ell$ both vanish.

This then gives us a new non-existence theorem for Einstein metrics:

Theorem 3.3 Let $X$ be a compact oriented 4-manifold with a non-trivial Seiberg-Witten invariant. Then

$$
M=X \# k \overline{\mathbb{C P}}_{2} \# \ell\left(S^{1} \times S^{3}\right)
$$

does not admit Einstein metrics if $k+4 \ell \geq \frac{1}{3}(2 \chi+3 \tau)(X)$.

Proof. We may assume that $(2 \chi+3 \tau)(X)>0$, since otherwise the result follows from the Hitchin-Thorpe inequality.

Now

$$
(2 \chi+3 \tau)(M)=\frac{1}{4 \pi^{2}} \int_{M}\left(\frac{s_{g}^{2}}{24}+2\left|W_{+}\right|_{g}^{2}-\frac{|\stackrel{\circ}{r}|^{2}}{2}\right) d \mu_{g}
$$

for any metric on $g$ on $M$. If $g$ is an Einstein metric, the trace-free part $\stackrel{\circ}{r}$ of the Ricci curvature vanishes, and we then have

$$
\begin{aligned}
(2 \chi+3 \tau)(X)-k-4 \ell & =(2 \chi+3 \tau)(M) \\
& =\frac{1}{4 \pi^{2}} \int_{M}\left(\frac{s_{g}^{2}}{24}+2\left|W_{+}\right|_{g}^{2}\right) d \mu_{g} \\
& \geq \frac{2}{3}(2 \chi+3 \tau)(X)
\end{aligned}
$$


by Proposition 3.2, with equality only if $k$ and $\ell$ both vanish. If $M$ carries an Einstein metric, it therefore follows that

$$
\frac{1}{3}(2 \chi+3 \tau)(X)>k+4 \ell
$$

The claim now follows by contraposition.

Specializing to the case of complex surfaces, we now have:

Corollary 3.4 Let $\left(X, J_{X}\right)$ be a minimal complex surface of general type, and let $\left(M, J_{M}\right)$ be obtained from $X$ by blowing up $k$ points. Then the smooth compact 4-manifold $M$ does not admit any Einstein metrics if $k \geq \frac{1}{3} c_{1}^{2}(X)$.

Example Let $X \subset \mathbb{C P}_{3}$ be a hypersurface of degree 6 . Since the canonical class on $X$ is twice the hyperplane class, $c_{1}^{2}(X)=2^{2} \cdot 6=24$. Corollary 3.4 therefore tells us that if we blow up $X$ at 8 points, the resulting 4 -manifold

$$
M=X \# 8 \overline{\mathbb{C P}}_{2}
$$

does not admit Einstein metrics.

Now let us compare the complex surface $M$ with the Horikawa surface $N$ obtained as a ramified double cover of $\mathbb{C P}_{1} \times \mathbb{C P}_{1}$ branched at a generic curve of bidegree $(6,12)$. Both of these simply connected complex surfaces have $c_{1}^{2}=16$ and $p_{g}=10$, and the underlying oriented 4 -manifolds therefore have $b_{+}=21$ and $b_{-}=93$. In particular, both have signature $\tau=-72 \not \equiv 0 \bmod$ 16 , so, by Rochlin's theorem, neither is spin. Thus $M$ and $N$ have isomorphic intersection forms by the Minkowski-Hasse classification, and are therefore homeomorphic by Freedman's theorem 13. However, $N$ has ample canonical line bundle, and so admits a Kähler-Einstein metric by Yau's theorem. Thus, although $M$ and $N$ are homeomorphic, one admits Einstein metrics, while the other doesn't.

If we instead start with a hypersurface $X \subset \mathbb{C P}_{3}$ of degree $\geq 7$, one can construct homeomorphic pairs $(M, N)$ for which the minimal complex surface sits well above the Noether line. Details are left to the interested reader. $\diamond$

Remark Corollary 3.4 is the direct descendant of an analogous result in [28], 
where, using only scalar curvature estimates, a similar conclusion was proved for $k \geq \frac{2}{3} c_{1}^{2}(X)$. It was later pointed out by Kotschick [22] that such a result alone suffices to imply the existence of homeomorphic pairs consisting of an Einstein manifold and a 4-manifold which does not admit Einstein metrics; however, the examples that arise by this method are quite complicated, and have huge $c_{1}^{2}$.

The intermediate step between [28] and Corollary 3.4 may be found in [30], where Seiberg-Witten estimates of Weyl curvature were first introduced. While crude by present standards, the method used there did lead to an obstruction when $k \geq \frac{25}{57} c_{1}^{2}(X)$, or about two-thirds of the way to the present result.

The fact that $S^{1} \times S^{3}$ handles can be added to a 4-manifold without losing Seiberg-Witten control of the scalar curvature was first observed by Petean [33], although Seiberg-Witten theory only enters his result in an indirect manner. The search for a direct Seiberg-Witten explanation of this phenomenon then led the author and del Rio [31, 11] to a discovery of the generalized Seiberg-Witten invariant used here. In fact, however, this turned out to merely be a re-discovery; quite different considerations had already led Ozsváth and Szabó [32 to develop a full-blown theory, described in a preprint written the previous month.

Let us now recall that the Hitchin-Thorpe inequality [19, 42, 6] asserts that if a compact oriented 4-manifold $M$ admits an Einstein metric, then

$$
\frac{|\tau(M)|}{\chi(M)} \leq \frac{2}{3} .
$$

Hitchin went on to observe that the converse is certainly false, because the argument shows in particular that a simply connected 4-manifold with $2 \chi(M)=-3 \tau(M)$ can admit an Einstein metric only if its a $K 3$ surface. Examples of 4-manifolds with $|\tau(M)| / \chi(M)<2 / 3$ which do not admit Einstein metrics were first constructed by Gromov [15], using his simplicial volume invariant; however, this method only works in the presence of an infinite fundamental group. In [28], simply connected examples were first constructed, using Seiberg-Witten estimates for the scalar curvature. Shortly thereafter, Sambusetti [35] showed that the entropy estimates of Besson-Courtois-Gallot [7] allow one to construct examples of arbitrary Euler characteristic and signature, but again with huge fundamental group; see Petean [33] and del Rio [11 for similar results. In the simply connected case, however, it remains 
to be seen whether $(\chi, \tau)$ can really be arbitrarily specified, subject to the obvious constraints $\chi \geq 2+|\tau|$ and $\chi \equiv \tau \bmod 2$. Indeed, even the following is open:

Question 3.5 For every $q \in(-1,1) \cap \mathbb{Q}$, are there smooth, compact simply connected 4-manifolds with $\tau / \chi=q$ which do not admit Einstein metrics?

Hitchin-Thorpe gives one a resoundingly affirmative answer in the range $\frac{2}{3} \leq|q|<1$. The present results allow us to improve this as follows:

Corollary 3.6 Let $q$ be a rational number with $\frac{8}{23} \leq|q|<1$. Then there are smooth, compact, simply connected 4-manifolds with $\tau / \chi=q$ which do not admit Einstein metrics.

Proof. For $m$ any even integer bigger than 17 million, Chen 10 has constructed a simply connected minimal complex surface $X$ of general type (in fact, a hyperelliptic fibration) with $\tau(X)=m$ and $\chi(X)=4 m$. If we now blow up such a surface at $k$ points, where $k \geq \frac{11}{3} m$, the resulting simply connected 4-manifold $M=X \# k \overline{\mathbb{C P}}_{2}$ does not admit Einstein metrics by Corollary 3.4. Now for this manifold we have

$$
\frac{\tau(M)}{\chi(M)}=\frac{5}{4+\frac{k}{m}}-1
$$

and the right-hand side sweeps out $\mathbb{Q} \cap\left(-1,-\frac{8}{23}\right]$ as $\frac{k}{m}$ ranges over $\mathbb{Q} \cap\left[\frac{11}{3}, \infty\right)$. This proves the claim for $q$ negative. The case of $q$ positive then follows by reversing orientation.

The Weyl estimates of 82 also have interesting ramifications for the theory of anti-self-dual 4-manifolds. Recall that an oriented Riemannian 4-manifold is said to be anti-self-dual if it satisfies the $W_{+} \equiv 0$, and that this condition is conformally invariant. Compact anti-self-dual manifolds exist in profusion. Indeed, Taubes [39] has shown that for any smooth compact orientable $X^{4}$, there is an integer $k_{0}$ such that $M=X \# k \overline{\mathbb{C P}}_{2}$ admits metrics with $W_{+}=0$ provided that $k \geq k_{0}$. In particular, if we blow up a symplectic 4-manifold with $b^{+}>1$ at enough points, we obtain a manifold which admits anti-selfdual metrics and also has a non-trivial Seiberg-Witten invariant. Let us now consider the scalar curvature of such manifolds. 
Lemma 3.7 Let $(M, g)$ be a compact anti-self-dual 4-manifold with a nonzero Seiberg-Witten invariant. Then

$$
\int_{M} s^{2} d \mu \geq 72 \pi^{2}\left(c_{1}^{+}\right)^{2}
$$

where $c_{1}^{+}$is again the self-dual part of the first Chern class of the relevant spinc structure. If equality holds, moreover, $g$ is an almost-Kähler metric of the type describe in Proposition 2.4.

Proof. If $W_{+} \equiv 0$, (10) becomes

$$
\int_{M} \frac{4}{9} s^{2} d \mu \geq 32 \pi^{2}\left(c_{1}^{+}\right)^{2}
$$

and the desired inequality is therefore an immediate consequence. Moreover, if equality holds, Proposition 2.4 applies, and $g$ is an almost-Kähler manifold with the relevant special properties.

Notice that this result may be used as a vanishing theorem. For example, if $c$ is a $\operatorname{spin}^{c}$ structure on a hyperbolic 4-manifold, the corresponding SeibergWitten invariant will vanish unless $\left(c_{1}^{+}\right)^{2} \leq \frac{8}{3} \chi$. Since the non-negativity of the virtual dimension of the Seiberg-Witten moduli space would require that $\left(c_{1}^{+}\right)^{2} \geq 2 \chi+\left|c_{1}^{-}\right|^{2}$, this would seem to lend some weak support to the conjecture that the Seiberg-Witten invariants of a hyperbolic 4-manifold are all zero. In any case, it also implies the following result:

Theorem 3.8 Let $X$ be a compact oriented 4-manifold with a non-trivial Seiberg-Witten invariant. Then

$$
M=X \# k \overline{\mathbb{C P}}_{2} \# \ell\left(S^{1} \times S^{3}\right)
$$

does not admit anti-self-dual Einstein metrics if $k+4 \ell \geq \frac{1}{4}(2 \chi+3 \tau)(X)$.

Proof. As in the proof of Proposition 3.2, there is a monopole class $c$ of $(M, H)$ with

$$
c_{1}(L)=c_{1}(X)-\sum_{j=1}^{k} E_{j},
$$


and such that

$$
\left(c_{1}^{+}\right)^{2} \geq(2 \chi+3 \tau)(X) .
$$

If $M$ carried an anti-self-dual Einstein metric, Lemma 3.7 would then tell us that

$$
\begin{aligned}
(2 \chi+3 \tau)(X)-k-4 \ell & =(2 \chi+3 \tau)(M) \\
& =\frac{1}{4 \pi^{2}} \int_{M} \frac{s^{2}}{24} d \mu \\
& \geq \frac{72 \pi^{2}}{96 \pi^{2}}\left(c_{1}^{+}\right)^{2} \\
& \geq \frac{3}{4}(2 \chi+3 \tau)(X),
\end{aligned}
$$

so that

$$
k+4 \ell \leq \frac{1}{4}(2 \chi+3 \tau)(X) .
$$

Moreover, equality could only hold if $k$ and $\ell$ both vanished for precisely the same reasons delineated in the proof of Theorem 3.3.

The result now follows by contraposition.

\section{Ricci Curvature Estimates}

One of the most striking aspects of Seiberg-Witten theory is that it allows one to calculate the precise value of the invariant

$$
\mathcal{I}_{s}(M)=\inf _{g} \int_{M} s_{g}^{2} d \mu_{g}
$$

for large classes of 4-manifolds $M$; for example, if $M$ is a complex surface of general type, and if $X$ is its minimal model, then [28]

$$
\inf _{g} \int_{M} s_{g}^{2} d \mu_{g}=32 \pi^{2} c_{1}^{2}(X) .
$$

However, one would also like to compute the corresponding infimum

$$
\mathcal{I}_{r}(M)=\inf _{g} \int_{M}\left|r_{g}\right|^{2} d \mu_{g}
$$


for the Ricci curvature $r$. Since

$$
\int|r|^{2} d \mu=\int\left(\frac{s^{2}}{4}+|\stackrel{\circ}{r}|^{2}\right) d \mu,
$$

where $\stackrel{\circ}{r}$ is the trace-free part of the Ricci curvature, we obviously have

$$
\mathcal{I}_{r}(M) \geq \frac{1}{4} \mathcal{I}_{s}(M)
$$

and the scalar curvature estimate obviously gives us some important information about this problem. Moreover, because Kähler-Einstein metrics saturate the lower bound for the scalar curvature, one actually gets equality in (16) if $M$ happens to be a minimal complex surface of general type. For nonminimal surfaces, however, the situation is dramatically different; indeed, even the relatively crude Weyl estimates of [30] allow one to deduce that, for any complex surface $M$ of general type,

equality holds in $(10) \Longleftrightarrow M$ is minimal.

Until now, however, non-trivial direct calculations of $\mathcal{I}_{r}$ have remained beyond the scope of the theory. Fortunately, we will now see that the estimates of $\$ 2$ are perfectly suited to this purpose. The key observation is the following:

Proposition 4.1 Let $(M, H)$ be a polarized smooth compact oriented 4manifold with monopole class $c$. Then every $H$-adapted metric satisfies

$$
\frac{1}{4 \pi^{2}} \int_{M}\left(\frac{s_{g}^{2}}{24}+\frac{1}{2}\left|W_{+}\right|_{g}^{2}\right) d \mu_{g} \geq \frac{1}{2}\left(c_{1}^{+}\right)^{2} .
$$

Moreover, equality holds iff $g$ is a Kähler metric of constant scalar curvature, and the spinc structure is the one determined by a g-compatible complex structure on $M$.

Proof. We again begin with inequality (13),

$$
\frac{2}{3}\|s\|+\frac{1}{3}\left\|\left(\sqrt{24}\left|W_{+}\right|\right)\right\| \geq 4 \sqrt{2} \pi\left|c_{1}^{+}\right|,
$$

but this time interpret the left-hand side as the dot product

$$
\left(\frac{2}{3}, \frac{\sqrt{2}}{3}\right) \cdot\left(\|s\|,\left\|\left(\sqrt{12}\left|W_{+}\right|\right)\right\|\right)
$$


in $\mathbb{R}^{2}$. Applying the Cauchy-Schwarz inequality, we then obtain

$$
\left(\left(\frac{2}{3}\right)^{2}+\left(\frac{\sqrt{2}}{3}\right)^{2}\right)^{1 / 2}\left(\int_{M}\left(s^{2}+12\left|W_{+}\right|^{2}\right) d \mu\right)^{1 / 2} \geq \frac{2}{3}\|s\|+\frac{1}{3}\left\|\left(\sqrt{24}\left|W_{+}\right|\right)\right\| .
$$

Thus

$$
\frac{2}{3} \int_{M}\left(s^{2}+12\left|W_{+}\right|^{2}\right) d \mu \geq 32 \pi^{2}\left(c_{1}^{+}\right)^{2}
$$

and

$$
\frac{1}{4 \pi^{2}} \int_{M}\left(\frac{s_{g}^{2}}{24}+\frac{1}{2}\left|W_{+}\right|_{g}^{2}\right) d \mu_{g} \geq \frac{1}{2}\left(c_{1}^{+}\right)^{2}
$$

as claimed.

If equality holds, our use of Cauchy-Schwarz forces

$$
\left(\frac{2}{3}: \frac{\sqrt{2}}{3}\right)=\left(\|s\|:\left\|\left(\sqrt{12}\left|W_{+}\right|\right)\right\|\right)
$$

which is to say that

$$
\int\left|W_{+}\right|^{2} d \mu=\int \frac{s^{2}}{24} d \mu
$$

In this case, we then have

$$
\int_{M} s_{g}^{2} d \mu_{g}=32 \pi^{2}\left(c_{1}^{+}\right)^{2}
$$

and it then follows [27] that the metric is Kähler, and has constant scalar curvature. Conversely, a Kähler surface with constant scalar curvature satisfies $s \equiv \sqrt{24}\left|W_{+}\right|$and $\int s^{2} d \mu=32 \pi^{2}\left(c_{1}^{+}\right)^{2}$, so that equality is attained for any such metric.

This then gives rise to a Ricci curvature estimate via Gauss-Bonnet formulæ :

Proposition 4.2 Let $(M, H)$ be a polarized smooth compact oriented 4manifold with monopole class $c$. Then every $H$-adapted metric satisfies

$$
\frac{1}{8 \pi^{2}} \int_{M}\left|r_{g}\right|^{2} d \mu_{g} \geq 2\left(c_{1}^{+}\right)^{2}-(2 \chi+3 \tau)(M),
$$

where $r$ denotes the Ricci curvature of $g$. 
Proof. Since (四) tells us that

$$
(2 \chi+3 \tau)(M)=\frac{1}{4 \pi^{2}} \int_{M}\left(\frac{s_{g}^{2}}{24}+2\left|W_{+}\right|_{g}^{2}-\frac{|\stackrel{\circ}{r}|^{2}}{2}\right) d \mu_{g},
$$

it follows that

$$
\frac{1}{8 \pi^{2}} \int_{M}|r|^{2} d \mu=\frac{1}{\pi^{2}} \int_{M}\left(\frac{s_{g}^{2}}{24}+\frac{1}{2}\left|W_{+}\right|_{g}^{2}\right) d \mu_{g}-(2 \chi+3 \tau)(M)
$$

for any metric on $g$ on $M$. The result now follows immediately from Proposition 4.1.

This observation supplies the key to the main result of this section:

Theorem 4.3 Let $X$ be any minimal complex surface of general type, and let $M=X \# k \overline{\mathbb{C P}}_{2} \# \ell\left(S^{1} \times S^{3}\right)$. Then

$$
\mathcal{I}_{r}(M)=\inf _{g} \int_{M}|r|^{2} d \mu=8 \pi^{2}\left(c_{1}^{2}(X)+k+4 \ell\right) .
$$

Proof. Since

$$
(2 \chi+3 \tau)(M)=c_{1}^{2}(X)-k-4 \ell,
$$

equation (17) tells us that it suffices to prove that

$$
\inf _{g} \frac{1}{4 \pi^{2}} \int_{M}\left(\frac{s_{g}^{2}}{24}+\frac{1}{2}\left|W_{+}\right|_{g}^{2}\right) d \mu_{g}=\frac{1}{2} c_{1}^{2}(X) .
$$

Now observe that the proof of Proposition 3.2 tells us that that, for every metric $g$ on $M$, there is a $\operatorname{spin}^{c}$ structure $c$ with non-zero Seiberg-Witten invariant such that

$$
\left(c_{1}^{+}\right)^{2} \geq(2 \chi+3 \tau)(X)=c_{1}^{2}(X) .
$$

Hence

$$
\frac{1}{4 \pi^{2}} \int_{M}\left(\frac{s_{g}^{2}}{24}+\frac{1}{2}\left|W_{+}\right|_{g}^{2}\right) d \mu_{g} \geq \frac{1}{2} c_{1}^{2}(X)
$$

for every metric $g$ on $M$. It follows that

$$
\inf _{g} \frac{1}{4 \pi^{2}} \int_{M}\left(\frac{s_{g}^{2}}{24}+\frac{1}{2}\left|W_{+}\right|_{g}^{2}\right) d \mu_{g} \geq \frac{1}{2} c_{1}^{2}(X) .
$$


To finish the proof, it suffices to produce a family of metrics $g_{\varepsilon}$ on $M$ such that

$$
\limsup _{\varepsilon \rightarrow 0^{+}} \frac{1}{4 \pi^{2}} \int_{M}\left(\frac{s_{g_{\varepsilon}}^{2}}{24}+\frac{1}{2}\left|W_{+}\right|_{g_{\varepsilon}}^{2}\right) d \mu_{g_{\varepsilon}} \leq \frac{1}{2} c_{1}^{2}(X) .
$$

To do this, let $\check{X}$ be the pluri-canonical model of $X$, which carries a KählerEinstein orbifold metric $\check{g}$ by an immediate generalization [21, 44] of the Aubin/Yau solution [3, 47] of the $c_{1}<0$ case of the Calabi conjecture. Now

$$
\int_{\check{X}}\left(\frac{s_{\check{g}}^{2}}{24}+\frac{1}{2}\left|W_{+}\right|_{\check{g}}^{2}\right) d \mu_{\check{g}}=\frac{1}{2} c_{1}^{2}(\check{X})=\frac{1}{2} c_{1}^{2}(X)
$$

because $\left|W_{+}\right|^{2} \equiv s^{2} / 24$ for any Kähler surface, whereas a Kähler-Einstein surface has Ricci form given by $s \omega / 4$, where $\omega$ is the Kähler form. Let $o_{1}, \ldots, o_{n}$ be the orbifold points of $\bar{X}$, if there are any. Each of these singularities is of type A-D-E, meaning that $o_{j}$ has a neighborhood is modeled on $\mathbb{C}^{2} / \Gamma_{j}$ for some discrete sub-group $\Gamma_{j}$ of $S U(2)$. The passage from $\check{X}$ to $X$ is accomplished by replacing each $o_{j}$ by a collection of $(-2)$-curves with intersections determined by the Dynkin diagram (Coxeter graph) of type A-D-E corresponding to $\Gamma_{j}$.

One can now construct smooth metrics on the minimal model $X$ by modifying the orbifold metric $\breve{g}$, without introducing substantial amounts of extra volume, Ricci curvature, or self-dual Weyl curvature. Indeed, for each $\Gamma_{j}$ there are [23] gravitational instanton metrics on the minimal resolution of $\mathbb{C}^{2} / \Gamma_{j}$, which is precisely obtained by replacing the origin with a set of $(-2)$ curves as above. These gravitational instanton metrics are Ricci-flat and anti-self-dual, and they closely approximate the Euclidean metric on $\mathbb{C}^{2} / \Gamma_{j}$, being of the form $\delta_{j k}+O\left(\varrho^{-4}\right)$ in the asymptotic region, where $\varrho$ is the Euclidean radius; moreover, the first and second derivatives of such a metric are of order $O\left(\varrho^{-5}\right)$ and $O\left(\varrho^{-6}\right)$, respectively, in these coordinates. Fix such a metric, and consider the family of gravitational instanton metrics $h_{\varepsilon}$ obtained by multiplying it by $\varepsilon^{4}$, and then making the homothetic change $x \mapsto \varepsilon^{2} x$ of asymptotic coordinates. The metric $h_{\varepsilon}$ is then uniformly $\delta_{j k}+O\left(\varepsilon^{8} \varrho^{-4}\right)$ on the complement of the ball of radius $\varepsilon^{2}$, with first and second derivatives $O\left(\varepsilon^{8} \varrho^{-5}\right)$ and $O\left(\varepsilon^{8} \varrho^{-6}\right)$, respectively. Take geodesic spray coordinates about each of the orbifold points $o_{j}$, so that $\check{g}$ takes the form $\delta_{j k}+O\left(\varrho^{2}\right)$ in these coordinates. Delete the $\varepsilon$ ball around each orbifold point, and replace it in 
the $\varrho \leq \varepsilon$ region of the corresponding gravitational instanton; and on the transitional annulus $\varrho \in[\varepsilon, 2 \varepsilon]$, consider the metric

$$
g_{\varepsilon}=f\left(\frac{\varrho}{\varepsilon}\right) h_{\varepsilon}+\left[1-f\left(\frac{\varrho}{\varepsilon}\right)\right] \check{g}
$$

where $f:[1,2] \rightarrow[0,1]$ is a fixed smooth function which is $\equiv 1$ near 1 and $\equiv 0$ near 2 . This gives us a family of metrics on $X$, the geometry of which will be analyzed in a moment.

However, what we really need is a metric on $M$ rather than on $X$, so we need to modify $\check{g}$ a bit more. Now $S^{1} \times S^{3}$ and $\overline{\mathbb{C P}}_{2}$ both admit anti-self-dual metrics of positive scalar curvature - namely, the obvious product metric on $S^{1} \times S^{3}$, and the Fubini-Study metric on the reverse-oriented complex projective plane $\overline{\mathbb{C P}}_{2}$. The complement of any point in $S^{1} \times S^{3}$ or $\overline{\mathbb{C P}}_{2}$ therefore admits an asymptotically flat metric with $s \equiv 0$ and $W_{+} \equiv 0$, namely the standard metric rescaled by the Yamabe Greens function of the appropriate point. One can then find asymptotic charts for these manifolds, parameterized by the complement of, say, the unit ball in $\mathbb{R}^{4}$, in which these metrics differ from the Euclidean metric by $O\left(\varrho^{-2}\right)$ and in which first and second partial derivatives are respectively $O\left(\varrho^{-3}\right)$ and $O\left(\varrho^{-4}\right)$. (Notice the fall-off rate is slower than in the previous case; these metrics have positive mass!) Again multiplying by $\varepsilon^{4}$, where $\varepsilon$ is to be viewed as an auxiliary parameter, and making a homothety as before, we thus get metrics on the complement of the $\varepsilon^{2}$ ball in $\mathbb{R}^{4}$ of the form $\delta_{j k}+O\left(\varepsilon^{4} \varrho^{-2}\right)$, with first and second partial derivatives of order $O\left(\varepsilon^{4} \varrho^{-3}\right)$ and $O\left(\varepsilon^{4} \varrho^{-4}\right)$, respectively. Again, let $h_{\varepsilon}$ denote the restriction of the resulting metric on the the $\varrho>2 \varepsilon$ regions of $k$ disjoint punctured copies of $\overline{\mathbb{C P}}_{2}$ and $\ell$ disjoint punctured copies of $S^{1} \times S^{3}$. Take geodesic spray coordinates around non-singular points $p_{1}, \ldots, p_{k}, q_{1}, \ldots, q_{\ell}$ of $\check{X}$, and delete $\varepsilon$-balls around each one, and define a metric $g_{\varepsilon}$ on $M$ as $h_{\varepsilon}$ when $\varrho<\varepsilon$, as $\check{g}$ when $\varrho>2 \varepsilon$, and as (18) on the transitional annuli $\varrho \in[\varepsilon, 2 \varepsilon]$. The sectional curvature of the metrics $g_{\varepsilon}$ are then uniformly bounded on the annuli $\varepsilon \leq \varrho \leq 2 \varepsilon$ as $\varepsilon \searrow 0$, while the volumes of these annuli are $O\left(\varepsilon^{4}\right)$ as $\varepsilon \rightarrow 0$. Since the metrics we have used to replace the $\varepsilon$-balls are all scalar-flat and anti-self-dual, we therefore have

$$
\frac{1}{4 \pi^{2}} \int_{M}\left(\frac{s_{g_{\varepsilon}}^{2}}{24}+\frac{1}{2}\left|W_{+}\right|_{g_{\varepsilon}}^{2}\right) d \mu_{g_{\varepsilon}} \leq C \varepsilon^{4}+\frac{1}{4 \pi^{2}} \int_{\check{X}}\left(\frac{s_{\check{g}}^{2}}{24}+\frac{1}{2}\left|W_{+}\right|_{\check{g}}^{2}\right) d \mu_{\check{g}},
$$

and it thus follows that

$$
\limsup _{\varepsilon \rightarrow 0^{+}} \frac{1}{4 \pi^{2}} \int_{M}\left(\frac{s_{g_{\varepsilon}}^{2}}{24}+\frac{1}{2}\left|W_{+}\right|_{g_{\varepsilon}}^{2}\right) d \mu_{g_{\varepsilon}} \leq \frac{1}{2} c_{1}^{2}(X) .
$$


The theorem follows.

Corollary 4.4 Let $M$ be the underlying 4-manifold of any compact complex surface of Kodaira dimension $\geq 0$. Let $X$ be the minimal model of $M$, and let $k$ be the number of points at which $X$ must be blown up so as to obtain M. Then

$$
\inf _{g} \int_{M}|r|^{2} d \mu=8 \pi^{2}\left(c_{1}^{2}(X)+k\right),
$$

where the infimum is taken over all smooth Riemannian metrics on $g$ on $M$.

Proof. Complex surfaces of Kodaira dimension 2, are, by definition, precisely those of general type; for these, the result is just the $\ell=0$ case of Theorem 4.3. On the other hand, the analogous assertion for Kodaira dimensions 0 and 1 immediately follows from the fact 29] that any elliptic surface admits sequences of metrics for which $\operatorname{Vol}(M, g) \searrow 0$, but for which $s$ and $\left|W_{+}\right|$are uniformly bounded.

In particular, in view of (14), this gives a satisfying new proof of the following:

Corollary 4.5 Let $M$ be the underlying 4-manifold of any complex surface of Kodaira dimension $\geq 0$. Then

$$
\text { equality holds in (10) } \Longleftrightarrow M \text { is minimal. }
$$

We are also see that the invariant $\mathcal{I}_{r}$ is extremely sensitive to changes in differentiable structure on a topological 4-manifold:

Corollary 4.6 Let $X$ and $\tilde{X}$ be two minimal simply connected complex surfaces with the same geometric genus $p_{g} \neq 0$, but with $c_{1}^{2}(X)-c_{1}^{2}(\tilde{X})=j>0$. Then for all positive integers $k$, the 4 -manifolds $\tilde{X} \# k \overline{\mathbb{C P}}_{2}$ and $X \#(j+k) \overline{\mathbb{C P}}_{2}$ are homeomorphic, but have different invariants $\mathcal{I}_{r}$.

Proof. The simply connected 4-manifolds $\tilde{X} \# k \overline{\mathbb{C P}}_{2}$ and $X \#(j+k) \overline{\mathbb{C P}}_{2}$ are non-spin and have the same invariants $b_{ \pm}$; Freedman's classification [13] therefore tells us that they are homeomorphic. On the other hand, because 
$p_{g} \neq 0$, these complex surfaces have Kodaira dimension $\geq 0$. Theorem 4.3 therefore tells us that

$$
\frac{1}{8 \pi^{2}} \mathcal{I}_{r}\left(\tilde{X} \# k \overline{\mathbb{C P}}_{2}\right)=c_{1}^{2}(\tilde{X})+k=c_{1}^{2}(X)-j+k
$$

whereas

$$
\frac{1}{8 \pi^{2}} \mathcal{I}_{r}\left(X \#(j+k) \overline{\mathbb{C P}}_{2}\right)=c_{1}^{2}(X)+(j+k) .
$$

Thus these manifolds have unequal invariants $\mathcal{I}_{r}$, as claimed.

Note that pairs $(X, \tilde{X})$ of the above kind are as common as garden weeds; cf. e.g. [5]. For example, if $X$ is of general type, we can always find a simply connected properly elliptic surface $\tilde{X}$ with the same geometric genus as $X$.

\section{Sectional Curvature and Volume}

In the previous section, we considered a differential-topological invariant arising out of the $L^{2}$-norm of Ricci curvature. We will now turn to a related problem which arises in connection with the $L^{\infty}$ norm of sectional curvature.

Recall that the sectional curvature $K(P)$ of a Riemannian $n$-manifold $(M, g)$ is a smooth function on the Grassmann bundle of 2-dimensional subspaces $P$ of $T M$. At each point $x \in M$, let us consider the bottom sectional curvature, given by

$$
\underline{K}(x):=\min _{P \subset T_{x} M} K(P) .
$$

Then $\underline{K}: M \rightarrow \mathbb{R}$ is automatically a Lipschitz continuous function, although it need not be differentiable in general. Notice that we tautologically have

$$
\underline{K} \leq \frac{s}{n(n-1)}
$$

so that $\underline{K}$ is negative on $\{x \in M \mid s(x)<0\}$.

Lemma 5.1 Let $(M, g)$ be an oriented Riemannian 4-manifold, and, for $x$ in $M$, let $w(x) \leq 0$ denote the smallest eigenvalue of $W_{+}(x): \Lambda_{x}^{+} \rightarrow \Lambda_{x}^{+}$. Then

$$
\underline{K}(x) \leq \frac{s(x)}{12}+\frac{w(x)}{2} .
$$

If equality holds at $x$, then $W_{-}(x)=0$; and the converse also holds if $g$ is Einstein. 
Proof. First recall that, in terms of the decomposition

$$
\Lambda^{2}=\Lambda^{+} \oplus \Lambda^{-}
$$

the curvature operator of an oriented Riemannian 4-manifold can be expressed in the form

$$
\mathcal{R}=\left(\begin{array}{c|c}
W_{+}+\frac{s}{12} & B \\
\hline B^{*} & W_{-}+\frac{s}{12}
\end{array}\right),
$$

where $W_{+}$and $W_{-}$are trace-free, and where $B: \Lambda^{-} \rightarrow \Lambda^{+}$exactly corresponds to $\stackrel{\circ}{r}$. On the other hand, every 2 -form $\psi$ on $M$ can be uniquely written as $\psi=\psi^{+}+\psi^{-}$, where $\psi^{ \pm} \in \Lambda^{ \pm}$. Now a 2 -form is expressible as a simple wedge product of 1 -forms iff $\psi \wedge \psi=0$. Thus $\psi$ is simple and has unit length iff $\left|\psi^{+}\right|^{2}=\left|\psi^{-}\right|^{2}=\frac{1}{2}$. The sectional curvature in the corresponding 2-plane is then

$$
K(P)=\frac{s}{12}+\left\langle\psi^{+}, W_{+}\left(\psi^{+}\right)\right\rangle+\left\langle\psi^{-}, W_{-}\left(\psi^{-}\right)\right\rangle+2\left\langle\psi^{+}, B \psi^{-}\right\rangle .
$$

Notice, however, that the last term changes sign if $\psi^{-}$is replaced by $-\psi^{-}$; geometrically, this means that the average of the sectional curvatures of an orthogonal pair of 2-planes only depends on the scalar and Weyl curvatures. Thus, there is always a 2-plane $P^{\prime}$ for which the sectional curvature is exactly

$$
K\left(P^{\prime}\right)=\frac{s}{12}+\left\langle\psi^{+}, W_{+}\left(\psi^{+}\right)\right\rangle+\left\langle\psi^{-}, W_{-}\left(\psi^{-}\right)\right\rangle .
$$

Hence

$$
\begin{aligned}
\underline{K} & \leq \frac{s}{12}+\inf _{\left|\psi^{+}\right|^{2}=\frac{1}{2}}\left\langle\psi^{+}, W_{+}\left(\psi^{+}\right)\right\rangle+\inf _{\left|\psi^{-}\right|^{2}=\frac{1}{2}}\left\langle\psi^{-}, W_{-}\left(\psi^{-}\right)\right\rangle \\
& =\frac{s}{12}+\frac{w}{2}+\frac{\tilde{w}}{2},
\end{aligned}
$$

where $\tilde{w} \leq 0$ is the lowest eigenvalue of $W_{-}$. In particular,

$$
\underline{K} \leq \frac{s}{12}+\frac{w}{2}
$$

as claimed. 
Proposition 5.2 Let $(M, g)$ be a compact oriented Riemannian 4-manifold on which there is a solution of the Seiberg-Witten equations. Let $c_{1}(L)$ be the first Chern class of the relevant spin ${ }^{c}$ structure, and let $c_{1}^{+}$denote its self-dual part with respect to $g$. Then

$$
-V^{1 / 2} \min _{x \in M}\left(\underline{K}(x)+\frac{s(x)}{12}\right) \geq \sqrt{2} \pi\left|c_{1}^{+}\right|
$$

where $V=\operatorname{Vol}(M, g)$ denotes the total volume of $(M, g)$.

Proof. By Proposition 2.1 we know that

$$
V^{1 / 3}\left(\int_{M}\left|\left(\frac{2}{3} s_{g}+2 w_{g}\right)_{-}\right|^{3} d \mu\right)^{2 / 3} \geq 32 \pi^{2}\left(c_{1}^{+}\right)^{2} .
$$

On the other hand,

$$
-4 \inf \left(\underline{K}_{g}+\frac{s_{g}}{12}\right) \geq\left(\frac{2}{3} s_{g}+2 w_{g}\right)_{-}
$$

at all points by virtue of $M$ by Lemma 5.1. Hence

$$
V^{1 / 3}\left(\int_{M}\left|4 \inf \left(\underline{K}_{g}+\frac{s_{g}}{12}\right)\right|^{3} d \mu\right)^{2 / 3} \geq 32 \pi^{2}\left(c_{1}^{+}\right)^{2},
$$

and so

$$
\left|\inf \left(\underline{K}_{g}+\frac{s_{g}}{12}\right)\right|^{2} V \geq 2 \pi^{2}\left(c_{1}^{+}\right)^{2} .
$$

Taking the square root of this inequality then yields the claim.

Let us now consider a natural family of minimal volume invariants 15 , 7, 28] defined with respect to only lower bounds on curvature. Let $M$ be any smooth compact manifold of dimension $n$. By just considering large constant multiples of some given metric, one obtains metrics on on $M$ with sectional curvature $K \geq-1$, at the price of making the total volume of $M$ enormous. This motivated Gromov [15] to define the Gromov minimal volume

$$
\operatorname{Vol}_{K}(M):=\inf \left\{\operatorname{Vol}(M, g) \mid K_{g} \geq-1\right\}
$$

as a way of quantifying the degree to which some negative sectional curvature might be an inevitable feature of all possible geometry on certain manifolds. 
However, it is also very natural to weaken the curvature hypothesis by instead only imposing lower bounds on the Ricci or scalar curvatures. In particular, one may consider [28] what we might call the Yamabe minimal volume of $M$, defined by

$$
\operatorname{Vol}_{s}(M):=\inf \left\{\operatorname{Vol}(M, g) \mid \frac{s_{g}}{n(n-1)} \geq-1\right\}
$$

where our conventions have been chosen so that, by definition,

$$
\operatorname{Vol}_{K}(M) \geq \operatorname{Vol}_{s}(M) \geq 0 \text {. }
$$

One key advantage of the latter definition is that the mature theory of the Yamabe problem [36] allows this minimal volume to be reinterpreted as

$$
\operatorname{Vol}_{s}(M)=\inf _{g} \int_{M}\left|\frac{s_{-}}{n(n-1)}\right|^{n / 2} d \mu_{g}=\left\{\begin{array}{cl}
0, & Y(M) \geq 0 \\
\left(\frac{|Y(M)|}{n(n-1)}\right)^{n / 2}, & Y(M) \leq 0
\end{array}\right.
$$

where $s_{-}=\min (s, 0)$, and where $Y(M)$ is the Yamabe invariant (sigma constant) of $M$.

It will now be convenient to consider an interpolation between these two definitions, gotten by averaging the two curvature conditions under consideration. We thus define the mixed minimal volume of a the smooth compact manifold $M$ to be

$$
\operatorname{Vol}_{K, s}(M)=\inf \left\{\operatorname{Vol}(M, g) \mid \frac{1}{2}\left(K_{g}+\frac{s_{g}}{n(n-1)}\right) \geq-1\right\} .
$$

This definition has been chosen in such a way that we now have the following result:

Theorem 5.3 Let $M$ be the underlying 4-manifold of any complex surface of general type. Then

$$
\operatorname{Vol}_{K, s}(M) \geq \frac{9}{4} \operatorname{Vol}_{s}(M)
$$

Moreover, equality holds if $M$ is any complex-hyperbolic manifold $\mathbb{C H}_{2} / \Gamma$, and in this case both minimal volumes are achieved by appropriate constant multiples of the standard metric. 
Proof. Let $X$ be the minimal model of $M$. Then for every metric $g$ on $M$ there is a $\operatorname{spin}^{c}$ structure with $\left(c_{1}^{+}\right)^{2} \geq c_{1}^{2}(X)$ and such that the corresponding Seiberg-Witten invariant is non-zero for the polarization determined by $g$; in particular, the scalar curvature $s$ of $g$ is negative somewhere. Thus, Proposition 5.2 tells us that $g$ satisfies

$$
V\left[\min _{x \in M}\left(\underline{K}(x)+\frac{s(x)}{12}\right)\right]^{2} \geq 2 \pi^{2}\left(c_{1}^{+}\right)^{2} \geq 2 \pi^{2} c_{1}^{2}(X) .
$$

Restricting our attention to metrics for which $\frac{1}{2}\left(\underline{K}+\frac{s}{12}\right) \geq-1$, we thus get

$$
\operatorname{Vol}_{K, s}(M) \geq \frac{2 \pi^{2} c_{1}^{2}(X)}{2^{2}}=\frac{\pi^{2}}{2} c_{1}^{2}(X) .
$$

However [28], $\operatorname{Vol}_{s}(M)=\frac{2 \pi^{2}}{9} c_{1}^{2}(X)$, so it follows that

$$
\operatorname{Vol}_{K, s}(M) \geq \frac{9}{4} \operatorname{Vol}_{s}(M)
$$

as claimed.

If $M$ is a complex-hyperbolic manifold, and if $g$ is a multiple of the hyperbolic metric, normalized so that its sectional curvatures satisfy $K \in\left[-\frac{1}{3},-\frac{4}{3}\right]$, one then has $s=-8$, and $\frac{1}{2}(\underline{K}+s) \equiv-1$, whereas $s^{2} V=32 \pi^{2} c_{1}^{2}(M)=$ $32\left(\frac{9}{2} \operatorname{Vol}_{s}(M)\right)$. Thus $V=\frac{9}{4} \operatorname{Vol}_{s}(M)$, and

$$
\operatorname{Vol}_{K, s}(M)=\frac{9}{4} \operatorname{Vol}_{s}(M)
$$

for any complex-hyperbolic 4-manifold.

Because of the tautological inequality

$$
\operatorname{Vol}_{K}(M) \geq \operatorname{Vol}_{K, s}(M),
$$

this immediately implies:

Corollary 5.4 If $M$ is any compact complex surface of general type, then

$$
\operatorname{Vol}_{K}(M) \geq \frac{9}{4} \operatorname{Vol}_{s}(M) .
$$


Of course, the constant appearing in this corollary should not be expected to be sharp. Indeed, inspection of the complex-hyperbolic case would instead seem to suggest the following:

Conjecture 5.5 If $M$ is any compact complex surface of general type, then

$$
\operatorname{Vol}_{K}(M) \geq 4 \operatorname{Vol}_{s}(M),
$$

with equality iff $M$ is complex hyperbolic.

Notice that we have not even shown that only the complex hyperbolic manifolds saturate the inequality of Theorem 5.3. Nonetheless, we can at least say the following:

Proposition 5.6 Suppose that $M$ is a complex surface of general type, and suppose that the mixed minimal volume $\operatorname{Vol}_{K, s}(M)$ is actually achieved by some metric $g$. Then either $\operatorname{Vol}_{K, s}(M)>\frac{9}{4} \operatorname{Vol}_{s}(M)$, or else $(M, g)$ is a complex-hyperbolic manifold, normalized so that $s \equiv-8$.

Proof. Suppose that $g$ is a metric with $K+\frac{s}{12} \geq-2$ and total volume $V=\frac{9}{4} \operatorname{Vol}_{s}(M)$. Thus, letting $X$ denote the minimal model of $M$, and choosing a $\operatorname{spin}^{c}$ structure on $M$ as in the proof of Proposition 3.2,

$$
\begin{aligned}
2 \pi^{2} c_{1}^{2}(X)=4 V & \geq V\left[\min \left(\underline{K}+\frac{s}{12}\right)\right]^{2} \\
& \geq \frac{1}{16} \int\left(\frac{2}{3} s+2 w\right)_{-}^{2} d \mu \\
& \geq \frac{32 \pi^{2}}{16}\left[c_{1}^{+}\right]^{2}=2 \pi^{2}\left[c_{1}^{+}\right]^{2}
\end{aligned}
$$

by virtue of (11). Since the $\operatorname{spin}^{c}$ structure is chosen so that $\left[c_{1}^{+}\right]^{2} \geq c_{1}^{2}(X)$, equality must hold, it follows that $c_{1}^{+}=c_{1}(X)$ and $c_{1}^{-}=0$; in particular, $M=X$ is minimal. Moreover, since (11) is saturated, Proposition 2.4 tells us that $g$ is almost-Kähler, with $s+s^{*}$ constant, $\omega$ an eigenvector of $W_{+}$ at each point, and $c_{1}^{+} \propto[\omega]$. Since $c_{1}=c_{1}^{+}$, the almost-complex structure satisfies $c_{1} \propto[\omega]$, so that our almost-Kähler manifold is monotonic in the terminology of [12].

Since equality holds in (22), we also have

$$
\underline{K}+\frac{s}{12} \equiv \frac{s}{6}+\frac{w}{2}
$$


and this, for starters, tells us that $W_{-} \equiv 0$ by (21). Inspection of (20) then shows that $B^{*}(\omega) \equiv 0$, so that $\omega$ is in fact an eigenvector of the full curvature operator $\mathcal{R}$. An almost-Kähler manifold with this property is called weakly *-Einstein. Because we also know that $(M, g, \omega)$ is monotonic, and that the sum $s+s^{*}$ of the scalar and $*$-scalar curvatures is constant, a result of Drăghici [12, Proposition 2] asserts that $(M, g, J)$ is Kähler-Einstein. But we also have also observed that $W_{-} \equiv 0$, so the entire curvature operator is parallel, and the universal cover of $(M, g)$ is therefore the symmetric space $\mathbb{C} \mathcal{H}_{2}$, equipped with the unique multiple of its standard metric for which $s=-8$. Thus $M$ admits a complex-hyperbolic metric; and this metric is moreover unique, up to rescalings and diffeomorphisms, by virtue of Mostow rigidity.

We now close with some final remarks concerning minimal volumes. First of all, one might want to consider more general mixed minimal volumes, where the scalar and sectional curvatures are weighted differently from above. It may therefore be worth noting that one can also show that

$$
\inf \left\{\operatorname{Vol}(M, g) \mid t K_{g}+(t-1) \frac{s}{12} \geq-1\right\} \geq(1+t)^{2} \operatorname{Vol}_{s}(M)
$$

for any complex algebraic surface $M$ and any constant $t \in\left[0, \frac{1}{2}\right]$; and again, equality holds for complex-hyperbolic manifolds. Indeed, it is not hard to establish inequalities such as

$$
\inf _{g} \int_{M}\left(\left(1-\frac{2 t}{3}\right) s+4 t w\right)_{-}^{2} d \mu \geq 32 \pi^{2}\left(c_{1}^{+}\right)^{2}
$$

for $t \in\left[0, \frac{1}{2}\right]$, and the assertion then follows. The interesting question, though, is whether such inequalities still hold for some $t>\frac{1}{2}$.

It would also be interesting to determine whether

$$
\operatorname{Vol}_{r}(M)=\inf \{\operatorname{Vol}(M, g) \mid r \geq-3 g\}
$$

is strictly larger than $\operatorname{Vol}_{s}(M)$ when $M$ is a non-minimal complex surface; our computations of $\mathcal{I}_{r}(M)$ certainly prove such an assertion for the related invariant

$$
\inf \{\operatorname{Vol}(M, g) \mid 3 g \geq r \geq-3 g\} \geq \frac{1}{36} \mathcal{I}_{r}(M),
$$

defined with respect to two-sided curvature bounds. Because the minimizing sequences constructed in the proof of Theorem 4.3 actually have huge 
amounts of Ricci curvature concentrated near the blow-ups, both of these minimal volumes will presumably turn out to be much larger than $\mathcal{I}_{r} / 36$ in the non-minimal case. A confirmation of this speculation would presumably also lead to even stronger non-existence theorems for Einstein metrics. And then, of course, the tautological inequality

$$
\operatorname{Vol}_{K}(M) \geq \operatorname{Vol}_{r}(M)
$$

would most pointedly inform us that our present estimates of the Gromov minimal volume have barely begun to scratch the surface of the subject.

\section{References}

[1] V. Apostolov, J. Armstrong, and T. Drăghici, Local Rigidity of Certain Classes of Almost Kähler 4-Manifolds, e-print math.DG/9911197, available at http://xxx.lanl.gov

[2] J. Armstrong, An Ansatz for Almost-Kähler, Einstein 4-Manifolds, Oxford preprint, 1999.

[3] T. Aubin, Equations du Type Monge-Ampère sur les Variétés Kählériennes Compactes, C. R. Acad. Sci. Paris 283A (1976) 119121.

[4] T. Aubin, Nonlinear Analysis on Manifolds. Monge-Ampère Equations, Springer-Verlag, 1982.

[5] W. Barth, C. Peters, and A. Van de Ven, Compact Complex Surfaces, Springer-Verlag, 1984.

[6] A. Besse, Einstein Manifolds, Springer-Verlag, 1987.

[7] G. Besson, G. Courtois, and S. Gallot, Entropies et Rigidités des Espaces Localement Symétriques de Courbure Strictement Négative, Geom. and Func. An. 5 (1995) 731-799.

[8] D. Blair, The 'Total Scalar Curvature' as a Symplectic Invariant, and Related Results, Proceedings of the 3rd Congress of Geometry (Thessaloniki, 1991), Aristotle Univ. Thessaloniki, 1992, pp. 79-83. 
[9] J.P. Bourguignon, Les Variétés de Dimension 4 à Signature Non Nulle dont la Courbure Est Harmonique Sont d'Einstein, Invent. Math. 63 (1981) 263-286.

[10] Z. Chen, On the Geography of Surfaces. Simply Connected Minimal Surfaces with Positive Index, Math. Ann. 277 (1987) 41-164.

[11] H. del Rio Guerra, Seiberg-Witten Invariants of Non-Simple Type and Einstein Metrics, e-print math.DG/0002243, available at http://xxx.lanl.gov

[12] T. Drăghici, On Some 4-Dimensional Almost Kähler Manifolds, Kodai Math. J. 18 (1995) 156-168.

[13] M. Freedman, On the Topology of 4-Manifolds, J. Diff. Geom. 17 (1982) 357-454.

[14] R. Fintushel and R. Stern, Immersed Spheres in 4-Manifolds and the Immersed Thom Conjecture, Turk. J. Math. 19 (1995) 145-157.

[15] M. Gromov, Volume and Bounded Cohomology, Publ. IHES 56 (1982) 5-99.

[16] M. Gromov, Pseudoholomorphic Curves in Symplectic Manifolds, Invent. Math. 82 (1985) 307-347.

[17] M. Gromov and H. B. Lawson, The Classification of Simply Connected Manifolds of Positive Scalar Curvature, Ann. of Math. 111 (1980) 423-434.

[18] M. Gursky, Four-Manifolds with $\delta W^{+}=0$ and Einstein Constants on the Sphere, Indiana University preprint, 1997.

[19] N. Hitchin, Compact Four-Dimensional Einstein Manifolds, J. Diff. Geom. 9 (1974) 435-441.

[20] E. Horikawa, Algebraic Surfaces of General Type with Small c $c_{1}^{2}$, I, Ann. Math. 104 (1976) 357-387.

[21] R. Kobayashi, Einstein-Kähler V-Metrics on Open Satake V-Surfaces with Isolated Quotient Singularities, Math. Ann. 272 (1985) 385-398. 
[22] D. Kotschick, Einstein Metrics and Smooth Structures, Geom. Topol. 2 (1998) 1-10.

[23] P. Kronheimer, Instantons Gravitationelles et Singularités de Klein, C.R. Acad. Sci. Paris 303 (1986) 53-55.

[24] P. Kronheimer and T. Mrowka, The Genus of Embedded Surfaces in the Complex Projective Plane, Math. Res. Lett. 1 (1994) 797-808.

[25] P. Kronheimer, Minimal Genus in $S^{1} \times M^{3}$, Invent. Math. 135 (1999) 45-61.

[26] C. LeBrun, Einstein Metrics and Mostow Rigidity, Math. Res. Lett. 2 (1995) 1-8.

[27] C. LeBrun, Polarized 4-Manifolds, Extremal Kähler Metrics, and Seiberg-Witten Theory, Math. Res. Lett. 2 (1995) 653-662.

[28] C. LeBrun, Four-Manifolds without Einstein Metrics, Math. Res. Lett. 3 (1996) 133-147.

[29] C. LeBrun, Kodaira Dimension and the Yamabe Problem, Comm. Anal. Geom. 7 (1999) 133-156.

[30] C. LeBrun, Weyl Curvature, Einstein Metrics, and Seiberg-Witten Theory, Math. Res. Lett. 5 (1998) 423-438.

[31] C. LeBrun, Four-Dimensional Einstein Manifolds, and Beyond, to appear in Essays on Einstein Manifolds, International Press, 2000.

[32] P. Ozsváth and Z. Szabó, Higher-Type Adjunction Inequalities in Seiberg-Witten Theory. Princeton University preprint, 1998.

[33] J. Petean, Computations of the Yamabe Invariant, Math. Res. Lett. 5 (1998) 703-709.

[34] J. Petean, Yamabe Invariants of Simply Connected Manifolds, e-print math.DG/9808062, available at http://xxx.lanl.gov

[35] A. Sambusetti, An Obstruction to the Existence of Einstein Metrics on 4-Manifolds, C. R. Acad. Sci. Paris 322 (1996) 1213-1218. 
[36] R. Schoen, Variational Theory for the Total Scalar Curvature Functional for Riemannian Metrics and Related Topics, Lec. Notes Math. 1365 (1987) 120-154.

[37] I. M. Singer and J. A. Thorpe, The Curvature of 4-dimensional Einstein Spaces, in Global Analysis (Papers in Honor of K. Kodaira), D. Spencer, eds, Univ. Tokyo Press, 1969, pp. 355-365.

[38] S. Stolz, Simply Connected Manifolds of Positive Scalar Curvature, Ann. of Math. 136 (1992) 511-540.

[39] C.H. Taubes, The Existence of Anti-Self-Dual Conformal Structures, J. Diff. Geom. 36 (1992) 163-253.

[40] C.H. Taubes, The Seiberg-Witten Invariants and Symplectic Forms, Math. Res. Lett. 1 (1994) 809-822.

[41] C.H. Taubes, The Seiberg-Witten and Gromov Invariants, Math. Res. Lett. 2 (1995) 221-238.

[42] J. Thorpe, Some Remarks on the Gauss-Bonnet Integral, J. Math. Mech. 18 (1969) 779-786.

[43] N. Trudinger, Remarks Concerning the Conformal Deformation of Metrics to Constant Scalar Curvature, Ann. Scuola Norm. Sup. Pisa 22 (1968) 265-274.

[44] H. Tsuji, Existence and Degeneration of Kähler-Einstein Metrics on Minimal Algebraic Varieties of General Type, Math. Ann. 281 (1988) 123-133.

[45] E. Witten, Monopoles and Four-Manifolds, Math. Res. Lett. 1 (1994) 809-822.

[46] H. Yamabe, On the Deformation of Riemannian Structures on Compact Manifolds, Osaka Math. J. 12 (1960) 21-37.

[47] S.-T. Yau, On the Ricci-Curvature of a Complex Kähler Manifold and the Complex Monge-Ampère Equations, Comment. Pure Appl. Math. 31 (1978) 339-411. 